FUNCTION SPACES XII

BANACH CENTER PUBLICATIONS, VOLUME 119

INSTITUTE OF MATHEMATICS

POLISH ACADEMY OF SCIENCES

WARSZAWA 2019

\title{
AN ALTERNATIVE TO PLANCHEREL'S CRITERION FOR BILINEAR OPERATORS
}

\author{
LOUKAS GRAFAKOS \\ Department of Mathematics, University of Missouri \\ Columbia MO 65211, USA \\ E-mail: grafakosl@missouri.edu
}

\begin{abstract}
We prove that bilinear operators associated with $L^{q}$ multipliers with sufficiently many derivatives in $L^{\infty}$ are bounded from $L^{2} \times L^{2}$ to $L^{1}$ when $q<4$. In the absence of Plancherel's identity on $L^{1}$, the range $q<4$ in the bilinear case should be compared to $q=\infty$ in the classical $L^{2} \rightarrow L^{2}$ boundedness for linear multiplier operators.
\end{abstract}

1. Introduction. Function spaces provide quantitative ways to measure integrability, smoothness, and to certain extent, cancellation properties of functions. A space of central importance is $L^{2}\left(\mathbb{R}^{n}\right)$ which appears at the crossroads of many echelons of function spaces. An important feature of $L^{2}\left(\mathbb{R}^{n}\right)$ is Plancherel's identity, which says that the Fourier transform

$$
\widehat{f}(\xi)=\lim _{N \rightarrow \infty} \int_{|x| \leq N} f(x) e^{-2 \pi i x \cdot \xi} d x \quad\left(\text { limit in } L^{2}\right)
$$

of a square-integrable function $f$ satisfies

$$
\|f\|_{L^{2}}=\|\widehat{f}\|_{L^{2}}
$$

(here $x \cdot y$ is the dot product on $\mathbb{R}^{n}$ ). This simply identity provides an alternative way to calculate $L^{2}$ norms. It also trivializes the characterization of the $L^{2}$-boundedness of convolution operators $\varphi \mapsto \varphi * K$, where $K$ is a tempered distribution. Plancherel's identity yields that such a convolution operator is bounded on $L^{2}\left(\mathbb{R}^{n}\right)$ if and only if the distributional Fourier transform of $K$ is a bounded function. Convolution operators can

2010 Mathematics Subject Classification: 42B15, 42B20, $42 \mathrm{~B} 99$.

Key words and phrases: Multiplier operators, bilinear operators, Plancherel's theorem. The paper is in final form and no version of it will be published elsewhere. 
also be expressed as multiplier operators. A multiplier operator has the form

$$
S_{m}(\varphi)(x)=\int_{\mathbb{R}^{n}} m(\xi) \widehat{\varphi}(\xi) e^{2 \pi i x \cdot \xi} d \xi
$$

where $m$ is a bounded function on $\mathbb{R}^{n}$ and is initially defined on Schwartz functions $\varphi$. We note that $S_{m}(\varphi)=\varphi * K$ whenever $\widehat{K}=m$. In view of Plancherel's identity we have

$$
\left\|S_{m}(f)\right\|_{L^{2}}=\left\|\widehat{S_{m}(f)}\right\|_{L^{2}}=\|m \widehat{f}\|_{L^{2}}
$$

and it follows from this that $S_{m}$ is $L^{2}$ bounded if and only if $m$ is an $L^{\infty}$ function. Moreover, the norm of $S_{m}$ from $L^{2}$ to itself is equal to $\|m\|_{L^{\infty}}$. This simple characterization of the $L^{2} \rightarrow L^{2}$ boundedness of multiplier operators is a direct consequence of Plancherel's identity, and for this reason we simply refer to it as Plancherel's criterion.

In this note we ask whether there exist boundedness criteria for bilinear translationinvariant operators analogous to Plancherel's criterion. Bilinear translation-invariant operators have the form

$$
T(f, g)(x)=\text { p.v. } \int_{\mathbb{R}^{n}} \int_{\mathbb{R}^{n}} K(x-y, x-z) f(y) g(z) d y d z, \quad x \in \mathbb{R}^{n},
$$

where $f, g$ are Schwartz functions and $K$ is a distribution on $\mathbb{R}^{2 n}$ that coincides with a suitable function on $\mathbb{R}^{2 n} \backslash\{(0,0)\}$. These operators can also be expressed as bilinear multiplier operators, i.e., operators of the form

$$
T_{m}(f, g)(x)=\int_{\mathbb{R}^{n}} \int_{\mathbb{R}^{n}} m(\xi, \eta) \widehat{f}(\xi) \widehat{g}(\eta) e^{2 \pi i x \cdot(\xi+\eta)} d \xi d \eta,
$$

initially defined for Schwartz functions $f, g$ where $m$ is a bounded function on $\mathbb{R}^{2 n}$. Note that $m$ coincides with the distributional Fourier transform of $K$. We refer to [4, Section 6 ] for general material related to the bilinear translation-invariant operators. These operators may map the product $L^{p_{1}}\left(\mathbb{R}^{n}\right) \times L^{p_{2}}\left(\mathbb{R}^{n}\right)$ to $L^{p}\left(\mathbb{R}^{n}\right)$ when $1 / p_{1}+1 / p_{2}=1 / p$ but in this note, we only focus on the $L^{2} \times L^{2} \rightarrow L^{1}$ boundedness of such operators. Such estimates are central and play the same role in bilinear theory as the $L^{2}$ boundedness plays in linear multiplier theory. As Plancherel's identity (1) does not hold on $L^{1}$, there does not seem to be a straightforward way to characterize the boundedness of bilinear multiplier operators from $L^{2} \times L^{2} \rightarrow L^{1}$. But for functions $m$ with bounded derivatives up to a certain order, such a characterization is possible.

As we restrict attention to multipliers all of whose derivatives are bounded, we introduce the space

$$
\mathcal{L}^{\infty}\left(\mathbb{R}^{2 n}\right)=\left\{m: \mathbb{R}^{2 n} \rightarrow \mathbb{C}: \partial^{\alpha} m \text { exist for all } \alpha \text { and }\left\|\partial^{\alpha} m\right\|_{L^{\infty}}<\infty\right\} .
$$

In the linear setting we have $m \in L^{\infty}$ if and only if the corresponding linear operator is bounded on $L^{2}$. So one may guess that a bilinear operator $T_{m}$ is bounded from $L^{2} \times L^{2}$ to $L^{1}$ when $m$ lies in $\mathcal{L}^{\infty}$. However Bényi and Torres [1] provided an example of a function $m \in \mathcal{L}^{\infty}$ for which the associated bilinear operator $T_{m}$ is unbounded from $L^{p_{1}} \times L^{p_{2}}$ to $L^{p}$ for any $1 \leq p_{1}, p_{2}<\infty$ satisfying $1 / p=1 / p_{1}+1 / p_{2}$. The counterexample of Bényi and Torres is also complemented by a subsequent positive result of He, Honzík, and the author [2, Corollary 8], who showed that the mere $L^{2}$ integrability of functions in $\mathcal{L}^{\infty}$ suffices to yield the $L^{2} \times L^{2} \rightarrow L^{1}$ boundedness of $T_{m}$. 
It turns out that the magnitude of integrability of a function $m$ in $\mathcal{L}^{\infty}$ characterizes the boundedness of the bilinear multiplier operator $T_{m}$ from $L^{2} \times L^{2} \rightarrow L^{1}$. We provide a proof of the main direction of this equivalence, the one that yields the boundedness of the operator.

THEOREM $1.1(\lfloor 3])$. Let $1 \leq q<4$ and set $M_{q}=\left\lfloor\frac{2 n}{4-q}\right\rfloor+1$. Let $m$ be a function in $L^{q}\left(\mathbb{R}^{2 n}\right) \cap \mathcal{C}^{M_{q}}\left(\mathbb{R}^{2 n}\right)$ satisfying

$$
\left\|\partial^{\alpha} m\right\|_{L^{\infty}} \leq C_{0}<\infty \quad \text { for all multiindices } \alpha \text { with }|\alpha| \leq M_{q} .
$$

Then there is a constant $C$ depending on $n$ and $q$ such that the bilinear operator $T_{m}$ with multiplier $m$ satisfies

$$
\left\|T_{m}\right\|_{L^{2} \times L^{2} \rightarrow L^{1}} \leq C C_{0}^{1-q / 4}\|m\|_{L^{q}}^{q / 4} .
$$

Additionally, we are aware of examples indicating that for any $q \geq 4$ there exist functions $m \in L^{q}\left(\mathbb{R}^{2 n}\right) \cap \mathcal{L}^{\infty}\left(\mathbb{R}^{2 n}\right)$ such that the associated operator $T_{m}$ does not map $L^{2} \times L^{2}$ to $L^{1}$; see [3] for $q>4$ and [5] for $q=4$. These counterexamples complement Theorem 1.1 and indicate its sharpness; as this note is based on the lecture of the author at the Function Spaces XII conference, we do not describe these counterexamples here.

2. Product-type wavelets. We plan to outline the proof of Theorem 1.1 . This is based on the product-type wavelet method initiated by He, Honzík and the author in [2]. Our approach here incorporates several crucial combinatorial improvements. For the sake of a simple and clear presentation, we prove Theorem 1.1 only in the case where $n=1$.

We recall some facts related to product-type wavelets that will be crucial in our approach of proving Theorem 1.1. For a fixed $M \in \mathbb{N}$ there exist real-valued compactly supported functions $\psi_{F}, \psi_{M}$ in $\mathcal{C}^{k}(\mathbb{R})$, called father wavelet and mother wavelet, respectively, that satisfy

$$
\left\|\psi_{F}\right\|_{L^{2}(\mathbb{R})}=\left\|\psi_{M}\right\|_{L^{2}(\mathbb{R})}=1
$$

and

$$
\int_{\mathbb{R}} x^{k} \psi_{M}(x) d x=0 \quad \text { for all } 0 \leq k \leq M .
$$

Then the family of functions

$$
\begin{aligned}
& \bigcup_{\mu_{1}, \mu_{2} \in \mathbb{Z}}\left\{\psi_{F}\left(x_{1}-\mu_{1}\right) \psi_{F}\left(x_{2}-\mu_{2}\right)\right\} \\
& \cup \bigcup_{\mu_{1}, \mu_{2} \in \mathbb{Z}} \bigcup_{\lambda=0}^{\infty}\left\{2^{\lambda / 2} \psi_{F}\left(2^{\lambda} x_{1}-\mu_{1}\right) 2^{\lambda / 2} \psi_{M}\left(2^{\lambda} x_{2}-\mu_{2}\right)\right\} \\
& \cup \bigcup_{\mu_{1}, \mu_{2} \in \mathbb{Z}} \bigcup_{\lambda=0}^{\infty}\left\{2^{\lambda / 2} \psi_{M}\left(2^{\lambda} x_{1}-\mu_{1}\right) 2^{\lambda / 2} \psi_{F}\left(2^{\lambda} x_{2}-\mu_{2}\right)\right\} \\
& \cup \bigcup_{\mu_{1}, \mu_{2} \in \mathbb{Z}} \bigcup_{\lambda=0}^{\infty}\left\{2^{\lambda / 2} \psi_{M}\left(2^{\lambda} x_{1}-\mu_{1}\right) 2^{\lambda / 2} \psi_{M}\left(2^{\lambda} x_{2}-\mu_{2}\right)\right\}
\end{aligned}
$$

forms an orthonormal basis of $L^{2}\left(\mathbb{R}^{2}\right)$. This result is due to Triebe $\bigsqcup^{1}$ and its proof can be found in Triebel [6].

\footnotetext{
${ }^{1}$ as confirmed by him during the Function Spaces XII conference.
} 
We denote by $\mathcal{J}$ the set of all pairs $(\lambda, G)$ such that either $\lambda=0$ and $G=(F, F)$, or $\lambda$ is a nonnegative integer and $G$ has the form $(F, M),(M, F)$, or $(M, M)$. For $(\lambda, G) \in \mathcal{J}$ and $\left(\mu_{1}, \mu_{2}\right) \in \mathbb{Z}^{2}$ we set

$$
\Psi_{\mu_{1}, \mu_{2}}^{\lambda, G}\left(x_{1}, x_{2}\right)=2^{\lambda / 2} \psi_{G_{1}}\left(2^{\lambda} x_{1}-\mu_{1}\right) 2^{\lambda / 2} \psi_{G_{2}}\left(2^{\lambda} x_{2}-\mu_{2}\right) .
$$

for $\left(x_{1}, x_{2}\right) \in \mathbb{R}^{2}$, where $G=\left(G_{1}, G_{2}\right)$ and $(\lambda, G) \in \mathcal{J}$.

The cancellation of wavelets is manifested in the following result.

Lemma 2.1. Let $M$ be a positive integer. Assume that $m \in \mathcal{C}^{M+1}$ is a function on $\mathbb{R}^{2}$ such that

$$
\sup _{|\alpha| \leq M+1}\left\|\partial^{\alpha} m\right\|_{L^{\infty}} \leq C_{0}<\infty .
$$

Then for $(\lambda, G) \in \mathcal{J}$ and $\left(\mu_{1}, \mu_{2}\right) \in \mathbb{Z}^{2}$ we have

$$
\left|\left\langle\Psi_{\mu_{1}, \mu_{2}}^{\lambda, G}, m\right\rangle\right| \leq C C_{0} 2^{-(M+2) \lambda},
$$

provided that $\psi_{M}$ has $M$ vanishing moments.

This lemma can be easily proved and is essentially a restatement of Lemma 7 in [2]. Note that if $G=(F, F)$ there is no cancellation, however, there is no decay claimed in (4), as $\lambda=0$ in this case.

3. Proof of Theorem 1.1. To prove the theorem we use the product type wavelets introduced in the previous section. We begin by fixing a large number $M$ to be determined later, which denotes the number of vanishing moments of the mother wavelet.

For $(\lambda, G) \in \mathcal{J}$ and $\mu \in \mathbb{Z}^{2}$ we denote the wavelet coefficient by

$$
b_{\mu}^{\lambda, G}=\left\langle\Psi_{\mu}^{\lambda, G}, m\right\rangle \text {. }
$$

By [7. Theorem 1.64] and by the fact that $L^{q}=F_{q, 2}^{0}$, we obtain

$$
\|m\|_{L^{q}\left(\mathbb{R}^{2}\right)} \approx\left\|\left(\sum_{(\lambda, G) \in \mathcal{J}} \sum_{\mu \in \mathbb{Z}^{2}}\left|b_{\mu}^{\lambda, G} 2^{\lambda} \chi_{Q_{\lambda \mu}}\right|^{2}\right)^{1 / 2}\right\|_{L^{q}\left(\mathbb{R}^{2}\right)},
$$

where $Q_{\lambda \mu}$ is the cube centered at $2^{-\lambda} \mu$ with sidelength $2^{1-\lambda}$.

Now, let us fix $(\lambda, G) \in \mathcal{J}$. For notational simplicity, we write $b_{\mu}$ instead of $b_{\mu}^{\lambda, G}$ in what follows. We also denote by $\tilde{Q}_{\lambda \mu}$ the cube centered at $2^{-\lambda} \mu$ with sidelength $2^{-\lambda}$. Since these cubes are pairwise disjoint in $\mu$ (for the fixed value of $\lambda$ ), the equivalence (5) yields

$$
\begin{aligned}
\|m\|_{L^{q}\left(\mathbb{R}^{2}\right)} & \gtrsim 2^{\lambda}\left\|\left(\sum_{\mu \in \mathbb{Z}^{2}}\left|b_{\mu}\right|^{2} \chi_{Q_{\lambda \mu}}\right)^{1 / 2}\right\|_{L^{q}\left(\mathbb{R}^{2}\right)} \geq 2^{\lambda}\left\|\left(\sum_{\mu \in \mathbb{Z}^{2}}\left|b_{\mu}\right|^{2} \chi_{\tilde{Q}_{\lambda \mu}}\right)^{1 / 2}\right\|_{L^{q}\left(\mathbb{R}^{2}\right)} \\
& =2^{\lambda}\left\|\sum_{\mu \in \mathbb{Z}^{2}}\left|b_{\mu}\right| \chi_{\tilde{Q}_{\lambda \mu}}\right\|_{L^{q}\left(\mathbb{R}^{2}\right)}=2^{\lambda(1-2 / q)}\left(\sum_{\mu \in \mathbb{Z}^{2}}\left|b_{\mu}\right|^{q}\right)^{1 / q} .
\end{aligned}
$$

If we set $b=\left(b_{\mu}\right)_{\mu \in \mathbb{Z}^{2}}$, the preceding sequence of inequalities yields

$$
\|b\|_{\ell^{q}} \leq C 2^{-\lambda(1-2 / q)}\|m\|_{L^{q}}
$$

Also, Lemma 2.1 implies that

$$
\|b\|_{\ell^{\infty}} \leq C C_{0} 2^{-\lambda(M+2)},
$$

where $M$ is the number of vanishing moments of $\psi_{M}$. 
We have an infinite $\times$ infinite matrix of wavelet coefficients indexed by $\mathbb{Z}^{2}$. To better organize these coefficients, define

$$
U_{r}=\left\{(k, l) \in \mathbb{Z} \times \mathbb{Z}=\mathbb{Z}^{2}: 2^{-r-1}\|b\|_{\ell^{\infty}}<\left|b_{(k, l)}\right| \leq 2^{-r}\|b\|_{\ell^{\infty}}\right\},
$$

where $r$ is a nonnegative integer. Also, we write $U_{r}$ as a union of the following two disjoint sets:

$$
\begin{aligned}
& U_{r}^{1}=\left\{(k, l) \in U_{r}: \operatorname{card}\left\{s:(k, s) \in U_{r}\right\} \geq K\right\} ; \\
& U_{r}^{2}=\left\{(k, l) \in U_{r}: \operatorname{card}\left\{s:(k, s) \in U_{r}\right\}<K\right\},
\end{aligned}
$$

where $K$ is a positive number to be determined. Thinking of $U_{r}$ an infinite $\times$ infinite matrix with integers entries, in this splitting, we placed in $U_{r}^{1}$ all columns of $U^{r}$ that have size greater than or equal to $K$ and in $U_{r}^{2}$ the remaining ones. We call $U_{r}^{1}$ the long columns of $U_{r}$ and $U_{r}^{1}$ the short columns. Let us define

$$
E=\left\{k \in \mathbb{Z}:(k, l) \in U_{r}^{1} \text { for some } l \in \mathbb{Z}\right\} .
$$

This set is exactly the set of projections of all long columns. Then

$$
(\operatorname{card} E) K\left[2^{-(r+1)}\|b\|_{\ell^{\infty}}\right]^{q} \leq \sum_{(k, l) \in U_{r}^{1}}\left|b_{(k, l)}\right|^{q} \leq\|b\|_{\ell^{q}}^{q},
$$

and therefore

$$
\operatorname{card} E \leq K^{-1}\left[2^{-(r+1)}\|b\|_{\ell^{\infty}}\right]^{-q}\|b\|_{\ell^{q}}^{q} .
$$

Having separated the wavelet coefficients in groups we proceed with the analysis of the sums of the decomposition associated to these groups. Given $(k, l) \in \mathbb{Z} \times \mathbb{Z}$, it follows from the definition of $\Psi_{(k, l)}^{\lambda, G}$ that $\Psi_{(k, l)}^{\lambda, G}$ can be written in the tensor product form

$$
\Psi_{(k, l)}^{\lambda, G}\left(x_{1}, x_{2}\right)=\omega_{1, k}\left(x_{1}\right) \omega_{2, l}\left(x_{2}\right)
$$

and

$$
\left\|\omega_{1, k}\right\|_{L^{\infty}} \approx\left\|\omega_{2, l}\right\|_{L^{\infty}}=2^{\lambda / 2}
$$

Define

$$
m^{r, 1}=\sum_{(k, l) \in U_{r}^{1}} b_{(k, l)} \Psi_{(k, l)}^{\lambda, G}=\sum_{(k, l) \in U_{r}^{1}} b_{(k, l)} \omega_{1, k} \omega_{2, l} .
$$

Let $\mathcal{F}^{-1}$ denote the inverse Fourier transform. Then

$$
\begin{aligned}
\left\|T_{m^{r, 1}}(f, g)\right\|_{L^{1}} & \leq\left\|\sum_{(k, l) \in U_{r}^{1}} b_{(k, l)} \mathcal{F}^{-1}\left(\omega_{1, k} \widehat{f}\right) \mathcal{F}^{-1}\left(\omega_{2, l} \widehat{g}\right)\right\|_{L^{1}} \\
& \leq \sum_{k \in E}\left\|\omega_{1, k} \widehat{f}\right\|_{L^{2}}\left\|\sum_{l:(k, l) \in U_{r}^{1}} b_{(k, l)} \omega_{2, l} \widehat{g}\right\|_{L^{2}} \\
& \leq C \sum_{k \in E}\left\|\omega_{1, k} \widehat{f}\right\|_{L^{2}} 2^{\lambda / 2} 2^{-r}\|b\|_{\ell^{\infty}}\|g\|_{L^{2}} \\
& \leq C\left(\sum_{k \in E} 1\right)^{1 / 2}\left(\sum_{k \in E}\left\|\omega_{1, k} \widehat{f}\right\|_{L^{2}}^{2}\right)^{1 / 2} 2^{\lambda / 2} 2^{-r}\|b\|_{\ell^{\infty}}\|g\|_{L^{2}} \\
& \leq C\left\{K^{-1 / 2}\left[2^{-(r+1)}\|b\|_{\ell^{\infty}}\right]^{-q / 2}\|b\|_{\ell^{q}}^{q / 2}\right\}\left\{2^{\lambda / 2} 2^{-r}\|b\|_{\ell^{\infty}}\right\} 2^{\lambda / 2}\|f\|_{L^{2}}\|g\|_{L^{2}},
\end{aligned}
$$

where we used estimate (8) and the property that the supports of the functions $\omega_{1, k}$ and $\omega_{2, l}$ have finite overlap. 
Now define

$$
m^{r, 2}=\sum_{(k, l) \in U_{r}^{2}} b_{(k, l)} \omega_{1, k} \omega_{2, l}
$$

Then

$$
\begin{aligned}
\left\|T_{m^{r, 2}}(f, g)\right\|_{L^{1}} & \leq\left\|\sum_{(k, l) \in U_{r}^{2}} b_{(k, l)} \mathcal{F}^{-1}\left(\omega_{1, k} \widehat{f}\right) \mathcal{F}^{-1}\left(\omega_{2, l} \widehat{g}\right)\right\|_{L^{1}} \\
& \leq \sum_{l: \exists k(k, l) \in U_{r}^{2}}\left\|\omega_{2, l} \widehat{g}\right\|_{L^{2}}\left\|\sum_{k:(k, l) \in U_{r}^{2}} b_{(k, l)} \omega_{1, k} \widehat{f}\right\|_{L^{2}} \\
& \leq\left(\sum_{l \in \mathbb{Z}}\left\|\omega_{2, l} \widehat{g}\right\|_{L^{2}}^{2}\right)^{1 / 2}\left(\sum_{l: \exists k(k, l) \in U_{r}^{2}} \sum_{k:(k, l) \in U_{r}^{2}} b_{(k, l)} \omega_{1, k} \widehat{f} \|_{L^{2}}^{2}\right)^{1 / 2} \\
& \leq C 2^{\lambda / 2}\|g\|_{L^{2}}\left(\sum_{k: \exists l(k, l) \in U_{r}^{2}}\left\|\omega_{1, k} \widehat{f}\right\|_{L^{2}}^{2} \sum_{l:(k, l) \in U_{r}^{2}}\left|b_{(k, l)}\right|^{2}\right)^{1 / 2} \\
& \leq C 2^{\lambda / 2}\|g\|_{L^{2}} 2^{-r}\|b\|_{\ell \infty} K^{1 / 2}\left(\sum_{k \in \mathbb{Z}}\left\|\omega_{1, k} \widehat{f}\right\|_{L^{2}}^{2}\right)^{1 / 2} \\
& \leq C 2^{\lambda / 2} 2^{-r}\|b\|_{\ell^{\infty}} K^{1 / 2} 2^{\lambda / 2}\|f\|_{L^{2}}\|g\|_{L^{2} .}
\end{aligned}
$$

We have now obtained the estimates for an unknown quantity $K$ :

$$
\begin{aligned}
& \left\|T_{\sigma_{1}^{r}}(f, g)\right\|_{L^{1}} \leq C K^{-1 / 2}\left[2^{-(r+1)}\|b\|_{\ell^{\infty}}\right]^{-q / 2}\|b\|_{\ell^{q}}^{q / 2} 2^{\lambda} 2^{-r}\|b\|_{\ell^{\infty}}\|f\|_{L^{2}}\|g\|_{L^{2}} \\
& \left\|T_{\sigma_{2}^{r}}(f, g)\right\|_{L^{1}} \leq C 2^{\lambda} 2^{-r}\|b\|_{\ell^{\infty}} K^{1 / 2}\|f\|_{L^{2}}\|g\|_{L^{2}} .
\end{aligned}
$$

We choose $K$ optimally so that the two quantities on the right above are equal. The optimal choice of $K$ is

$$
K=\left(\frac{2^{r}\|b\|_{\ell^{q}}}{\|b\|_{\ell^{\infty}}}\right)^{q / 2}
$$

which yields for

$$
m^{r}=\sum_{(k, l) \in U_{r}} b_{(k, l)} \omega_{1, k} \omega_{2, l}=m^{r, 1}+m^{r, 2}
$$

the estimate

$$
\left\|T_{m^{r}}\right\|_{L^{2} \times L^{2} \rightarrow L^{1}} \leq C 2^{\lambda} 2^{-r(1-q / 4)}\|b\|_{\ell^{\infty}}^{1-q / 4}\|b\|_{\ell^{q}}^{q / 4} .
$$

Using (6) and (7) we obtain

$$
\left\|T_{m^{r}}\right\|_{L^{2} \times L^{2} \rightarrow L^{1}} \leq C C_{0}^{1-q / 4} 2^{\lambda-\lambda(1-q / 4)(M+2)+(2 / q-1) \lambda q / 4} 2^{-r(1-q / 4)}\|m\|_{L^{q}}^{q / 4} .
$$

But

$$
2^{\lambda-\lambda(1-q / 4)(M+2)+(2 / q-1) \lambda q / 4}=2^{\lambda[1 / 2-((4-q) / 4)(M+1)]}
$$

and the exponent is negative only when $M+1>\frac{2}{4-q}$. Thus, if we choose $M=\left\lfloor\frac{2}{4-q}\right\rfloor$, we can sum first over $r$ and then over $(\lambda, G)$ in $\mathcal{J}$, obtaining (3). This completes the proof of Theorem 1.1

Acknowledgments. Research of the author was supported by the Simons Foundation.

This note is based on the lecture given by the author at the Function Spaces XII meeting held at the Jagiellonian University in Kraków, Poland during the week of 
July 9-14, 2018. The author would like to take this opportunity to thank the organizing committee of the conference, especially Grzegorz Lewicki and Mieczysław Mastyło, for the invitation, hospitality, and the assistance they provided him with during the meeting.

\section{References}

[1] A. Bényi, R. H. Torres, Almost orthogonality and a class of bounded bilinear pseudodifferential operators, Math. Res. Lett. 11 (2004), 1-11.

[2] L. Grafakos, D. He, P. Honzík, Rough bilinear singular integrals, Adv. Math. 326 (2018), 54-78.

[3] L. Grafakos, D. He, L. Slavíková, $L^{2} \times L^{2} \rightarrow L^{1}$ boundedness criteria, Math. Ann., to appear, DOI: $10.1007 / \mathrm{s} 00208-018-1794-5$.

[4] L. Grafakos, R. H. Torres, Multilinear Calderón-Zygmund theory, Adv. Math. 165 (1999), 124-164.

[5] L. Slavíková, Personal communication.

[6] H. Triebel, Bases in Function Spaces, Sampling, Discrepancy, Numerical Integration, EMS Tracts Math. 11, Eur. Math. Soc., Zürich, 2010.

[7] H. Triebel, Theory of Function Spaces III, Monogr. Math. 100, Birkhäuser, Basel, 2006. 
